\title{
Reduced-port robotic total mesorectal resection for rectal cancer using a single-port access: a technical note
}

\author{
Sung Uk Bae, Woon Kyung Jeong, Seong Kyu Baek \\ Keimyung University, Dongsan Medical Center, Daegu, South Korea
}

Videosurgery Miniinv 2017; 12 (4): 378-384

DOI: https://doi.org/10.5114/wiitm.2017.69727

\begin{abstract}
Introduction: Single-port laparoscopic surgery has some advantages, including improved cosmetic outcomes and minimized parietal trauma. However, pure single-port laparoscopic rectal cancer surgery is challenging because of the difficulties in creating triangulation and applying the laparoscopic staplers with sufficient distal margins in the narrow pelvic cavity. Recently, a reduced-port robotic operation with a robotic single-port access plus one wristed robotic arm for colon cancer was introduced to overcome the limitations of single-port laparoscopic rectal surgery.

Aim: Single-port laparoscopic surgery has some advantages, including improved cosmetic outcomes and minimized parietal trauma. However, the pure single-port laparoscopic rectal cancer operation is challenging. Recently, a reduced-port robotic operation with a robotic single-port access plus one wristed robotic arm for colon cancer was introduced to overcome the limitations of single-port laparoscopic rectal surgery.

Material and methods: We performed a single-port plus an additional port robotic operation using a robotic single-port access through the umbilical incision, and the wristed robotic instruments were inserted through an additional robotic port in the right lower quadrant.

Results: The total operative and docking times were $310 \mathrm{~min}$ and $25 \mathrm{~min}$, respectively. The total number of lymph nodes harvested was 12, and the proximal and distal resection margins were 11.1 and $2 \mathrm{~cm}$, respectively. The patient was discharged on postoperative day 12 uneventfully.

Conclusions: Based on a representative case, reduced-port robotic total mesorectal excision for rectal cancer using the single-port access appears to be feasible and safe. This approach could overcome the limitations of single-port laparoscopic rectal surgery.
\end{abstract}

Key words: rectal neoplasms, robotic surgical procedures, laparoscopy, natural orifice endoscopic surgery, lymph node excision.

\section{Introduction}

In rectal cancer surgery, the critical consideration for rectal resection is a sharp dissection that yields complete or total mesorectal excision (TME) [1]. Since the first laparoscopic TME for rectal neoplasm was described, there have been several multi-center, prospective, randomized studies about the efficacy of laparoscopic rectal operation for rectal cancer [2-4].
However, laparoscopic TME is regarded as technically demanding; because the rectum is located concavely along the curved sacrum in a narrow bony pelvic cavity, laparoscopic in-line rigid instruments have technical limitations.

Single-port laparoscopic surgery (SPLS) has some advantages, including improved cosmetic outcomes and minimized parietal trauma. Since the first reports on colectomy for malignant diseases through

\section{Address for correspondence}

Seong Kyu Baek MD, Department of Surgery, School of Medicine, Dongsan Medical Center, Keimyung University, 194 Dongsan-Dong,

Jung-Gu, 700-712 Daegu, South Korea, phone: +82 53250 8050, fax: +82 53250 7322, e-mail: sgbeak@dsmc.or.kr 
a transumbilical single-port access were published in 2010 [5], the worldwide popularity of this technique has rapidly increased, and some surgeons have adopted this technique to perform laparoscopic procedures, including rectal operation [6-14]. However, pure SPLS for rectal cancer is a challenging procedure, although the clinicopathologic results of pure SPLS for rectal cancer seem acceptable in previous retrospective case series because of the difficulties in creating triangulation and applying the laparoscopic staplers with sufficient distal margins in the narrow pelvic cavity.

Recently, reduced-port robotic surgery with robotic single-port access plus one wristed robotic arm for colon cancer was introduced to overcome the limitations of the single-port laparoscopic rectal operation $[15,16]$.

\section{Aim}

Herein, we describe the first case of reduced-port robotic total mesorectal resection for rectal cancer using the single-port access.

\section{Surgical technique}

\section{Representative case}

A 78-year-old woman with rectal cancer had a biopsy-proven adenocarcinoma within the rectum.
A colonoscopy revealed a $2-\mathrm{cm}$ sized ulcerofungating mass within $7 \mathrm{~cm}$ of the anal verge (Photo $1 \mathrm{~A}$ ). The pelvic magnetic resonance imaging scan showed an ulcerofungating mass in the rectum, and the radionuclide positron emission tomography scan of the torso (basal skull to the proximal thigh) using 18F-fluorodeoxyglucose showed a hypermetabolic lesion in the rectum without any distant metastasis (Photo $1 \mathrm{~B}$ ). The preoperative clinical stage was determined as CT1NOMO. We planned to perform a reduced-port robotic approach for rectal cancer using the Da Vinci Single-Site platform (Intuitive Surgical, Sunnyvale, CA).

\section{Technique}

The patient underwent total robotic total mesorectal excision using the Da Vinci Single-Site platform with the dual-docking technique (Figure 1). In this study, the colonic stage included lymphovascular dissection around the root of the inferior mesentery artery (IMA) with autonomic nerve preservation and colonic mobilization, whereas the pelvic stage included TME and intracorporeal end-to-end anastomosis.

\section{Installation and docking of the robotic platform}

The operation was performed under general anesthesia with the patient in the lithotomy position.
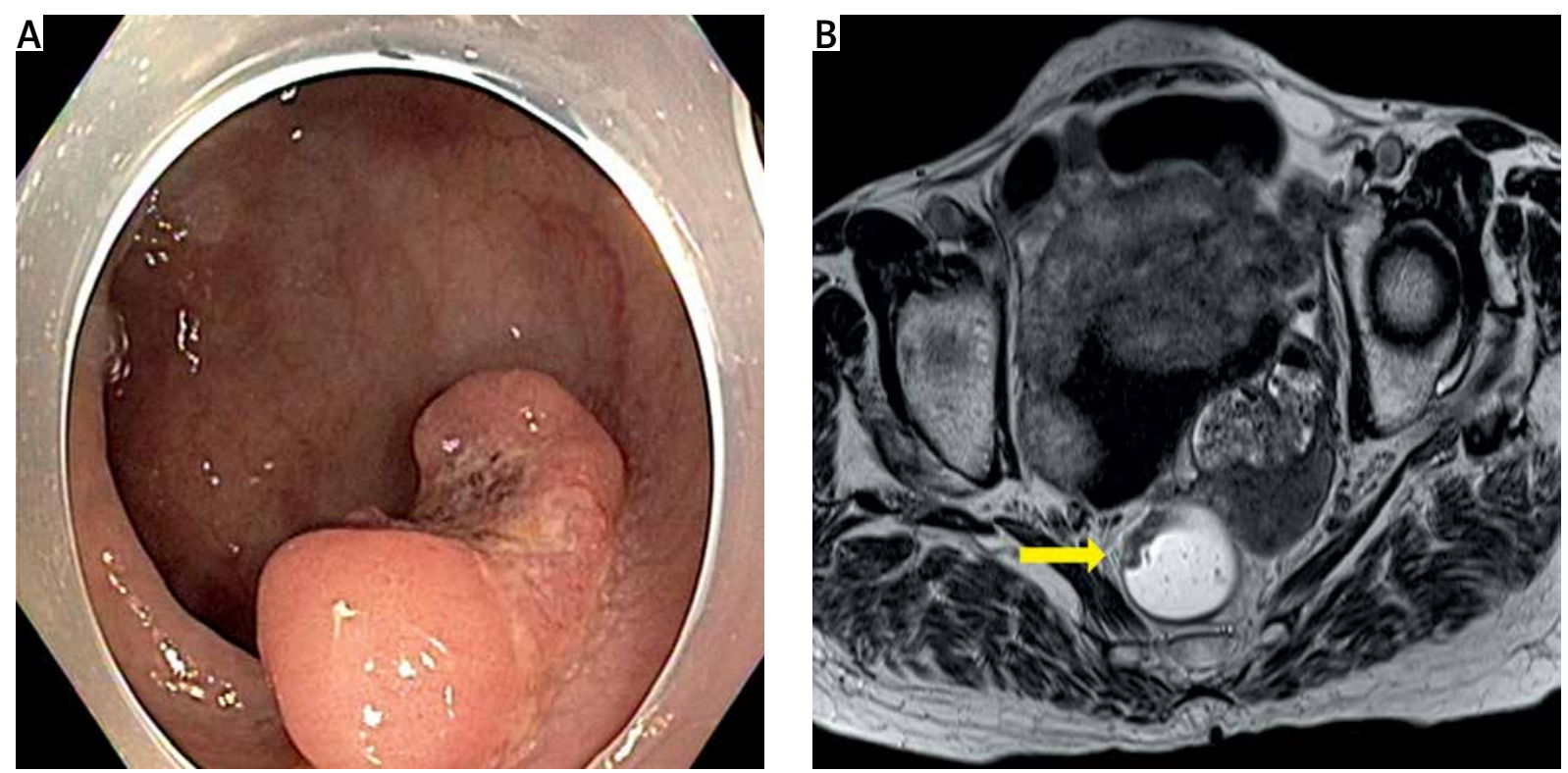

Photo 1. A - Colonoscopy shows a 2-cm ulcerofungating mass within $7 \mathrm{~cm}$ of the anal verge, B - the pelvic magnetic resonance imaging scan shows an ulcerofungating mass in the rectum 


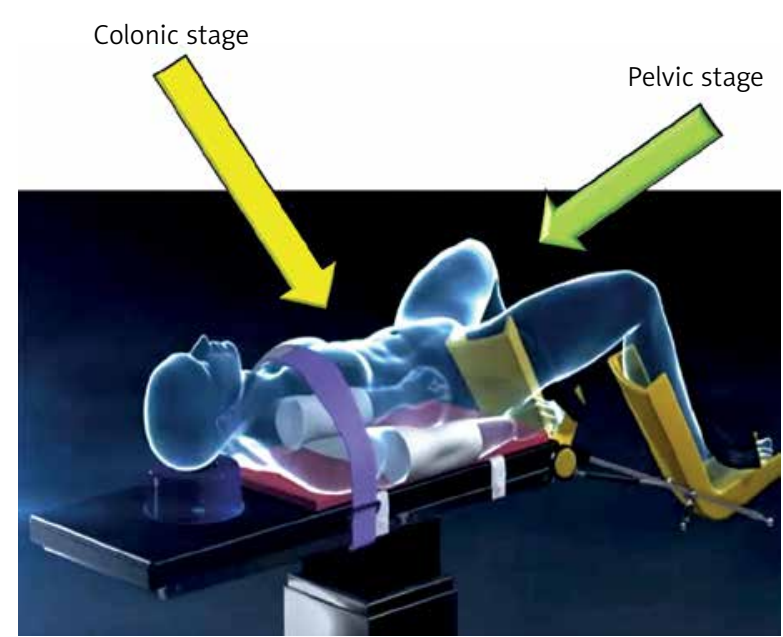

Figure 1. Dual-docking technique during reduced-port robotic total mesorectal excision for rectal cancer

A single $3.5-\mathrm{cm}$ transumbilical vertical incision was made, and the Glove port (Nelis, Bucheon, Korea) was inserted into the intra-abdominal space (Figure 2). After pneumoperitoneum with insufflations of $\mathrm{CO}_{2}$ to $12 \mathrm{~mm} \mathrm{Hg}$ was made, an $8.5-\mathrm{mm}$ robotic endoscope with a $30^{\circ}$ angled view was inserted. An additional single port for the wristed robotic instruments and rectal retraction was inserted in the right lower quadrant (Figure 2). The patient was placed in the Trendelenburg position at $30^{\circ}$ and tilted right side down at an angle of $15^{\circ}$, and the patient cart was positioned over the left flank, approaching the patient at an angle of approximately $90^{\circ}$. The camera arm was docked to the corresponding cannula, and the robotic endoscope was reinserted and attached to the camera arm. The two $5 \times 250-\mathrm{mm}$ crisscrossed curved cannulas were lubricated and inserted under direct visualization through the single port, and the R1 and R2 arms were docked to the

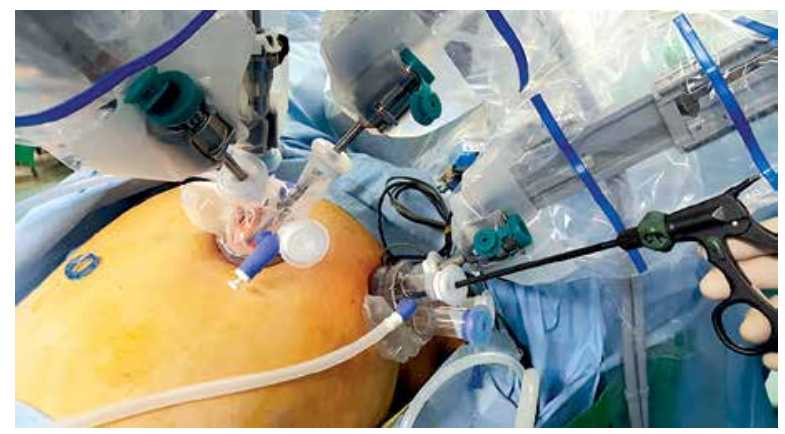

Photo 2. Access port setup for reduced-port robotic rectal operation

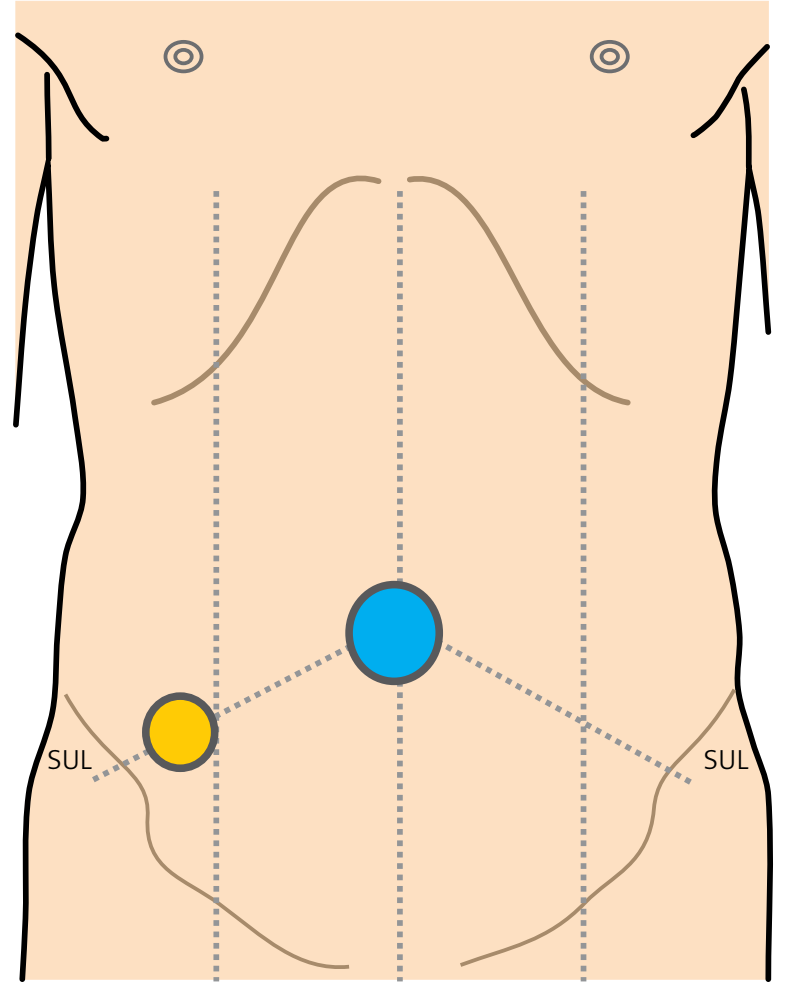

Figure 2. Port placement for reduced-port robotic rectal operation

robotic platform (Photo 2). The R3 arm was docked to the $8-\mathrm{mm}$ conventional robotic port through an additional single port in the right lower quadrant. To secure the distance between the tip of the instruments and the surgical field, especially lymph node dissection for inferior mesenteric vessels, the remote centers of R1 and R2 cannulas and the camera port were lifted upward out of the abdominal wall, and all three remote centers were aligned at the same height. The R1 arm was used for the Maryland Dissector (Novare Surgical Systems, Inc., Cupertino, (A), which was available for bipolar coagulation; the R2 arm was used for the Cadiere Forcep (EndoWrist; Intuitive Surgical, Sunnyvale, CA); and the R3 arm was used for the wristed monopolar curved scissor, EndoWrist One Vessel Sealer (Intuitive Surgical), and robotic endo-stapler.

\section{Stage 1: colonic phase}

The robotic second arm of the Single-Site platform was used to lift up the sigmoid pedicle; the peritoneum was incised at the level of the sacral promontory; and medial-to-lateral dissection was commenced along the right common iliac artery 
to expose the superior hypogastric plexus, ureter, and sigmoid artery (Photo $3 \mathrm{~A}$ ). Dissection of lymph nodes around the root of the IMA and high ligation of the IMA, with preservation of the autonomic nerve, was performed (Photo $3 \mathrm{~B}$ ). After the inferior mesenteric vein was lifted up by the second robotic arm, dissection between the surgical plane of the left Toldt fascia and retroperitoneal structures, including the gonadal vessels, Gerota fascia, and left ureter, was performed (Photo $3 \mathrm{C}$ ). The left paracolic gutter was dissected up to the spleen, although complete splenic mobilization, including dissection of the root of the transverse colon free from the lower border of the pancreas and detachment of the greater omentum of the transverse colon, was not performed (Photo $3 \mathrm{D}$ ).
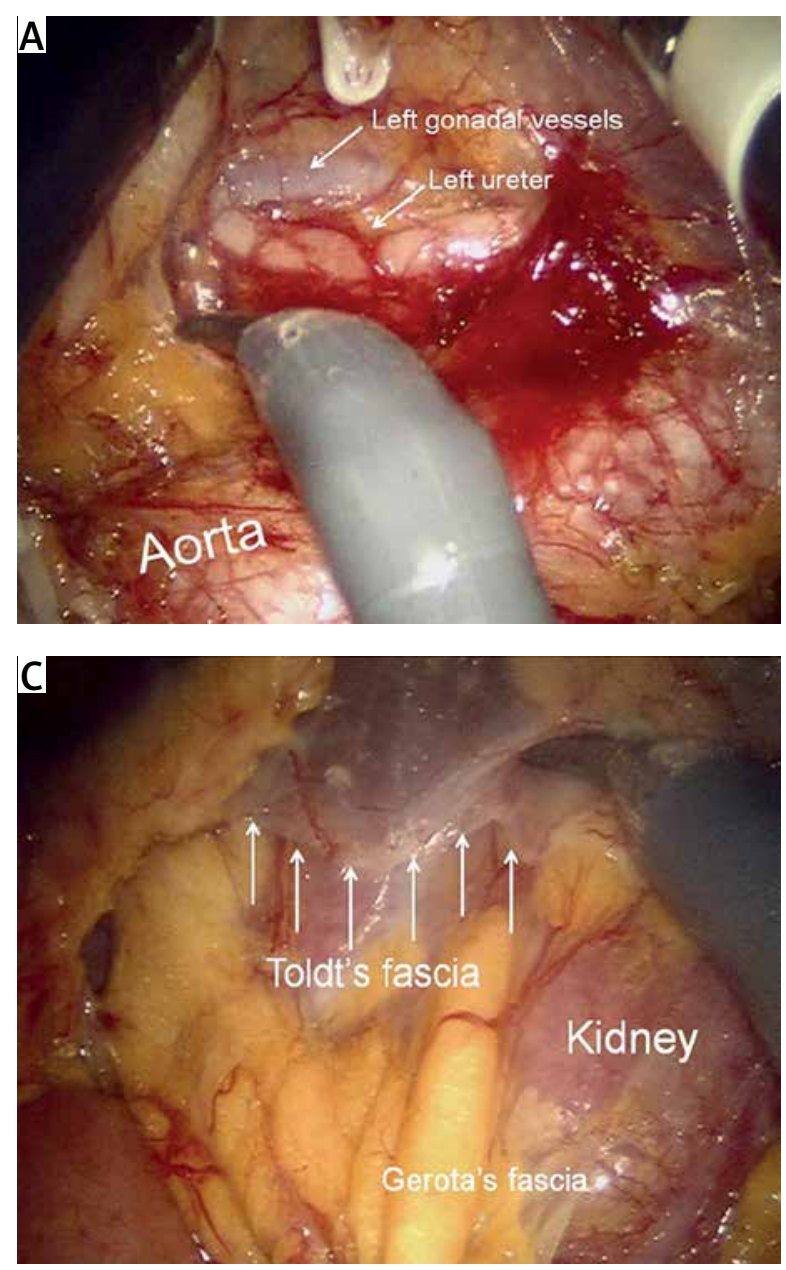

Stage 2: pelvic phase

After completing the colonic stage, the robotic cart was repositioned between the patient's legs, and the two $5 \times 250-\mathrm{mm}$ curved cannulas were replaced with two $5 \times 300-\mathrm{mm}$ curved cannulas for deep pelvic dissection. During stage 2 , the assistant surgeon used the additional single port to retract the rectosigmoid cephalad. Pelvic dissection was performed following the principles of TME and autonomic nerve preservation (Photos $4 \mathrm{~A}-\mathrm{C}$ ). For distal bowel resection, two 45-mm EndoWrist Staplers (Intuitive Surgical) were used through the additional single port in the right lower quadrant (Photo 4 D). The tumor specimen was extracted through the transumbilical incision, and end-to-end intracorporeal anastomosis
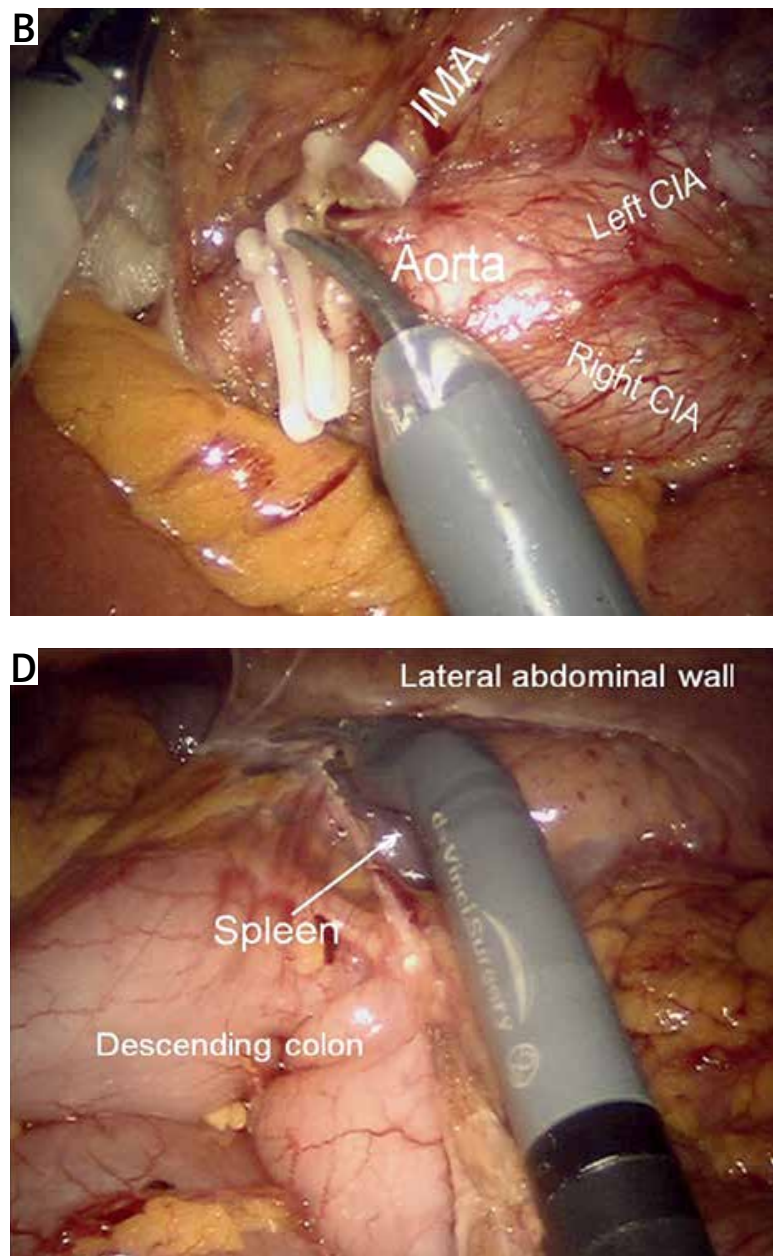

Photo 3. Reduced-port robotic total mesorectal excision for rectal cancer in the colonic phase. A - Medial-to-lateral dissection of the sigmoid colon. B - High ligation of the inferior mesenteric artery, with preservation of the autonomic nerve. $\mathbf{C}$ - Dissection between the surgical plane of the left Toldt fascia and retroperitoneal structures. D - Dissection of the left paracolic gutter up to the spleen 

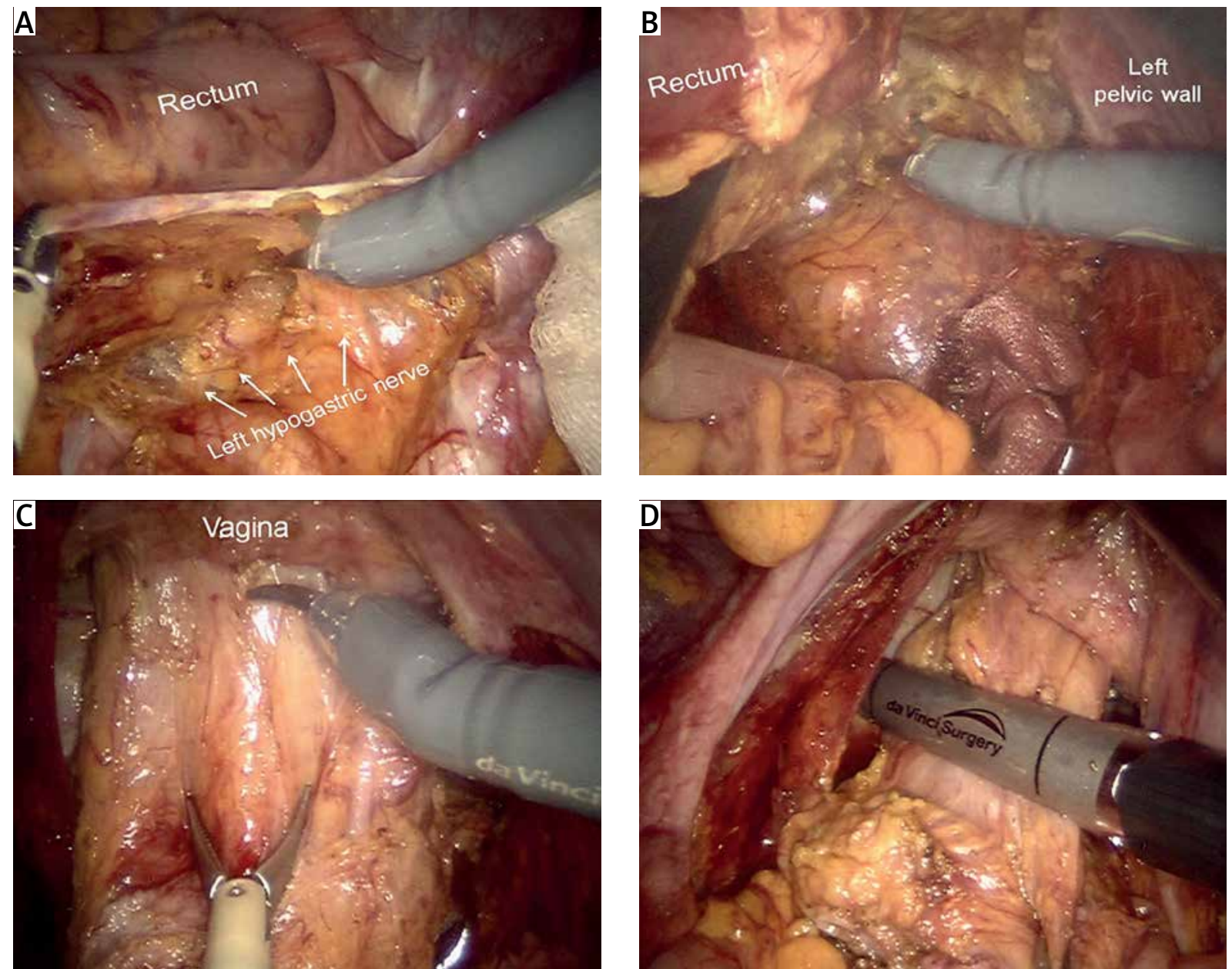

Photo 4. Reduced-port robotic total mesorectal excision for rectal cancer in the pelvic phase. A - Posterior rectal dissection. B - Lateral rectal dissection. C - Anterior rectal dissection. D - Endostapling using the robotic stapler through the additional port

was conducted with a double-stapling technique. The additional single port site was used for suction drain placement in the pelvic cavity. The anastomosis was then tested through an air leak test.

\section{Clinicopathologic outcomes}

A reduced-port totally robotic approach using a modified dual-docking technique facilitated lymphovascular dissection around the IMA and TME with minimal external instrument conflicts. The procedure was successfully completed without conversion to laparoscopic or open operation, and the total operative and docking times were $310 \mathrm{~min}$ and 25 min, respectively. The total incision length was $60 \mathrm{~mm}$. The patient developed postoperative pseudomembranous colitis that required conservative treatment. She was discharged on postoperative day 12 uneventfully. The pathologic examination of the specimen showed a $1.7 \times 1.2-\mathrm{cm}$ moderately differentiated T1NOMO adenocarcinoma without circumferential margin involvement. The total number of lymph nodes harvested was 12 , and the proximal and distal resection margins were 11.1 and $2 \mathrm{~cm}$, respectively.

\section{Discussion}

In this patient, we performed reduced-port robotic total mesorectal resection for rectal cancer using a single-port access to maintain the advantages of the robotic system (three-dimensional magnified vision, wristed instrumentation, stable camera platform, tremor filtration and single-port operation, better cosmesis, and decreased parietal trauma) and to overcome the limitations of the single-port 
laparoscopic rectal operation (lack of triangulation with straight instruments, ergonomic discomfort, and difficulty in stapling in the narrow pelvic cavity). The procedure was safely and feasibly completed without conversion to an open, laparoscopic, or multi-port robotic approach or compromising the pathologic outcomes, although the patient developed a minor complication that required conservative treatment.

Hamzaoglu et al. [6] reported the first human cases of single-port laparoscopic sphincter-saving mesorectal excision for rectal cancer using the double-stapling technique in 2 patients and hand-sewn anastomosis in 1 patient. Bulut et al. [9] reported the short-term outcomes of SPLS for rectal cancer in 25 patients. The median operating time was $260 \mathrm{~min}$, and the median postoperative stay was 7 days. The median number of harvested lymph nodes was 13 , and the surgical margins were clear in all patients.

Some authors have modified the SPLS technique to include the use of an additional port placed through the eventual drain site in the right lower quadrant. This approach may decrease collisions between the laparoscopic instruments and the camera, while maintaining the cosmetic advantages of a single-incision laparoscopic operation, making it possible to transect the lower rectum with pelvic drain placement [10, 11]. Since we first used the Single-Site platform to perform colorectal cancer operation in August 2014, we have modified this technique for laparoscopic stapling of the rectum, wristed instrumentation, and pelvic drain placement [16-18]. Additionally, in the present patient, the robotic stapler was used though the additional port to facilitate rectal resection in the narrow pelvic cavity using fully wristed articulation and smart clamp feedback that minimizes the guesswork associated with conventional stapling.

The distance between the tip of the robotic instruments and the surgical field is important, because even 250-mm curved cannulas are too long for performing dissection around the aortic bifurcation and inferior mesenteric vessels. In our previous study, we lifted the remote centers of R1 and R2 cannulas and the camera port upward out of the abdominal wall to shorten the distance between the tip of the robotic instruments and the surgical field, and to secure the surgical space [18]. In this study, during the pelvic stage, the two $5 \times 250-\mathrm{mm}$ curved cannulas were replaced with two $5 \times 300-\mathrm{mm}$ curved cannulas to perform deep pelvic dissection. We be- lieve that the single-port robotic approach using the Da Vinci Single-Site system could be improved in the future by developing various lengths of cannulas that are not yet available.

In the current patient, we used a two single-port technique that has never been reported in the literature, as far as we know. Some authors have added a 12-mm additional port that was useful for introducing the stapler and decreasing conflict between the operative instruments and camera [8, 10, 11]. In our previous studies, an additional $8-\mathrm{mm}$ conventional robotic port was inserted in the right lower quadrant; however, in this patient, we inserted an additional single port for the wristed robotic instruments and to ensure more efficient rectal retraction by the laparoscopic instrument through the $2.5-\mathrm{cm}$ incision in the right lower quadrant $[16,17]$. We believe that continuous traction and counter-traction of the rectum are important surgical techniques for precise TME.

\section{Conclusions}

Based on the present case, reduced-port robotic TME for rectal cancer using a single-port access seems feasible and safe. This approach could overcome the limitations of the single-port laparoscopic rectal operation.

\section{Conflict of interest}

The authors declare no conflict of interest.

\section{References}

1. Heald RJ, Moran BJ, Ryall RD, et al. Rectal cancer: the Basingstoke experience of total mesorectal excision, 1978-1997. Arch Surg 1998; 133: 894-9.

2. Bonjer HJ, Deijen CL, Haglind E. A randomized trial of laparoscopic versus open surgery for rectal cancer. N Engl J Med 2015; 373: 194.

3. Jeong SY, Park JW, Nam BH, et al. Open versus laparoscopic surgery for mid-rectal or low-rectal cancer after neoadjuvant chemoradiotherapy (COREAN trial): survival outcomes of an open-label, non-inferiority, randomised controlled trial. Lancet Oncol 2014; 15: 767-74.

4. van der Pas MH, Haglind E, Cuesta MA, et al. Laparoscopic versus open surgery for rectal cancer (COLOR II): short-term outcomes of a randomised, phase 3 trial. Lancet Oncol 2013; 14: 210-8.

5. Podolsky ER, Curcillo PG $2^{\text {nd }}$. Single port access (SPA) surgery: a 24-month experience. J Gastrointest Surg 2010; 14: 759-67.

6. Hamzaoglu I, Karahasanoglu T, Baca B, et al. Single-port laparoscopic sphincter-saving mesorectal excision for rectal cancer: report of the first 4 human cases. Arch Surg 2011; 146: 75-81. 
7. Bulut $\mathrm{O}$, Nielsen CB, Jespersen N. Single-port access laparoscopic surgery for rectal cancer: initial experience with 10 cases. Dis Colon Rectum 2011; 54: 803-9.

8. Hirano Y, Hattori M, Douden K, et al. Single-incision plus one port laparoscopic anterior resection for rectal cancer as a reduced port surgery. Scand I Surg 2012; 101: 283-6.

9. Bulut O, Aslak KK, Rosenstock S. Technique and short-term outcomes of single-port surgery for rectal cancer: a feasibility study of 25 patients. Scand I Surg 2014; 103: 26-33.

10. Bae SU, Baek SJ, Min BS, et al. Reduced-port laparoscopic surgery for a tumor-specific mesorectal excision in patients with colorectal cancer: initial experience with 20 consecutive cases. Ann Coloproctol 2015; 31: 16-22.

11. Jung KU, Yun SH, Cho YB, et al. Single incision and reduced port laparoscopic low anterior resection for rectal cancer: initial ex perience in 96 cases. ANZ I Surg 2016; 86: 403-7.

12. Choi BJ, Lee SC, Kang WK. Single-port laparoscopic total mesorectal excision with transanal resection (transabdominal transanal resection) for low rectal cancer: initial experience with 22 cases. Int J Surg 2013; 11: 858-63.

13. Dumont F, Goere D, Honore C, Elias D. Transanal endoscopic total mesorectal excision combined with single-port laparoscopy. Dis Colon Rectum 2012; 55: 996-1001.

14. Sourrouille I, Dumont F, Goere D, et al. Resection of rectal cancer via an abdominal single-port access: short-term results and comparison with standard laparoscopy. Dis Colon Rectum 2013; 56: 1203-10.

15. Bae SU, Jeong WK, Bae OS, Baek SK. Reduced-port robotic anterior resection for left-sided colon cancer using the Da Vinci single-site platform. Int J Med Robot 2015; 12: 517-23.

16. Bae SU, Jeong WK, Baek SK. Robotic anterior resection for sigmoid colon cancer using reduced port access. Dis Colon Rectum 2016; 59: 245-6.

17. Bae SU, Jeong WK, Bae OS, Baek SK. Reduced-port robotic anterior resection for left-sided colon cancer using the Da Vinci single-site(R) platform. Int J Med Robot 2015; 12: 517-23.

18. Bae SU, Jeong WK, Baek SK. Current status of robotic single-port colonic surgery. Int I Med Robot 2017; 13

Received: 25.05.2017, accepted: 10.07.2017. 